\title{
Nitrito urinário e infecção do trato urinário por cocos gram-positivos
}

\author{
Urinary nitrite and urinary-tract infection by gram-positive cocci
}

Andréa de Fátima Sato'; Arthur Estivalet Svidzinski; Marcia Edilaine Lopes Consolaro3; ${ }^{3}$ Cinthia Gandolfi Boer ${ }^{3}$

Uniterm
Infecção do trato uri
Tiras reagentes
Nitrito
Cocos gram-positivo

\section{resumo}

Introdução: A infecção do trato urinário (ITU) é uma das mais comuns na clínica médica. Os testes que utilizam tiras reagentes detectam significativamente mais infecções por bactérias gram-negativas do que por espécies gram-positivas, uma vez que o teste de nitrito não revela a presença de patógenos gram-positivos em muitos casos. Objetivos: Explorar a microbiologia e a freqüência de infecção urinária por cocos em adultos sintomáticos, relacionando-as com o resultado de nitrito urinário, para traçar o perfil epidemiológico desses pacientes. Material e método: Um estudo retrospectivo foi conduzido na Secretaria de Saúde do município de Maringá-PR, no período de abril de 2004 a março de 2005. Resultados: Amostras de urina de 3.426 pacientes foram coletadas e analisadas. Um total de $448(13,1 \%)$ amostras foi positivo para cultura. Em $388(86,6 \%)$ casos houve crescimento de bacilos gram-negativos (BGN) e em $60(13,4 \%)$ casos, cocos gram-positivos (CGP). A análise estatística de co-morbidades revelou diferença significativa na proporção de pacientes com hipertensão $(31,3 \%$ com nitrito positivo versus $4,5 \%$ com nitrito negativo, $p<0,05)$. Os pacientes com resultado de nitrito positivo relataram febre em proporção maior que os com nitrito negativo ( $75 \%$ versus $43,2 \%$, respectivamente; $p<0,05$ ). Staphylococcus aureus redutores de nitrato mostraram maior resistência à ciprofloxacina $(66,7 \%)$ quando em comparação com os isolados não-redutores (100\% sensíveis). Conclusões: Este estudo preliminar mostrou que a avaliação de sinais clínicos e sintomas, juntamente com o resultado de nitrito urinário na tira reagente, poderia auxiliar o médico na tomada de decisões importantes.
Background: Urinary tract infection (UTI) is among the most common infections in medical center. Urinalysis tests detect significantly more gram-negative infections than those due to gram-positive bacteria because the urinary nitrite test does not detect the presence of gram-positive pathogens in many cases. Objectives: Explore the microbiology and frequency of UTI by coccus in symptomatic adult patients, comparing them with urinary nitrite results, in order to delineate the epidemiological profile of these patients. Material and method: Retrospective analysis was conducted from April 2004 through March 2005 at Health Center, Maringa, Brazil. Results: Urine specimens from 3,426 patients were collected and examined. A total of $448(13.1 \%)$ samples had positive culture results. There were 388 (86.6\%) strains of gram-negative rods and 60 (13.4\%) of grampositive cocci. A statistical analysis of patients' comorbidities revealed a significant difference in the proportion of patients with hypertension (31.3\% with positive nitrite vs. $4.5 \%$ with negative nitrite, $p<0.05$ ). The proportion of patients with positive nitrite who had fever was larger than patients with negative nitrite (75\% vs. $43.2 \%$ respectively, $p<0.05)$. Nitrate-reducing Staphylococcus aureus showed high level resistance to ciprofloxacin (66.7\%) in comparison with nitrite-negative isolates (100\% sensitive). Conclusions: This preliminary study had shown that evaluation of clinical signs and symptoms, together with urinary nitrite result, could help physician to take important decisions. key words

Urinary tract infection

Dipsticks

Nitrites

Gram-positive cocci

1. Farmacêutica-bioquímica do Laboratório Municipal de Análises Clínicas de Maringá; aluna do curso de especialização em Análises Clínicas da Universidade Estadual de Maringá (UEM).

2. Aluno do curso de Farmácia-Bioquímica da UEM.

3. Professoras-adjuntas do Departamento de Análises Clínicas da UEM.

Trabalho baseado em monografia de especialização apresentada na UEM. Estudo realizado no Laboratório Municipal de Análises Clínicas da Secretaria de Saúde do Município de Maringá-PR. 


\section{Introdução}

A infecção do trato urinário (ITU) é uma das mais comuns na clínica médica, sendo definida como a invasão microbiana de qualquer órgão do trato urinário desde a uretra até os rins $^{(14)}$. O processo infeccioso pode afetar o rim, a pelve renal, os ureteres, a bexiga e a uretra, bem como as estruturas adjacentes, incluindo próstata e epidídimo, existindo possibilidades de agravamento na dependência do estado geral do paciente e da sua idade, merecendo, assim, grande atenção em pesquisas e estudos nas ciências médicas ${ }^{(11)}$. Podem ocorrer com ou sem sintomas, nesse caso sendo conhecido como bacteriúria assintomática ${ }^{(9)}$.

As ITU acometem homens e mulheres em qualquer idade, sendo que os grupos mais comprometidos são recém-nascidos do sexo masculino, meninas em idade pré-escolar, mulheres jovens sexualmente ativas, homens com obstrução prostática e idosos de ambos os $\operatorname{sexos}^{(3)}$. Após o primeiro ano de vida, as infecções tornam-se mais comuns no sexo feminino. A atividade sexual e a gravidez favorecem a infecção urinária ${ }^{(11)}$.

O trato urinário pode ser invadido por uma grande diversidade de microrganismos, como bactérias, fungos e vírus ${ }^{(6)}$. A natureza do microorganismo invasor depende, na maior parte dos casos, da história da infecção, dos fatores subjacentes do hospedeiro (anomalias congênitas ou fatores obstrutivos ao longo do aparelho urinário), do uso de agentes antimicrobianos e da instrumentação do trato urinário. A maioria das infecções urinárias é causada por bactérias gram-negativas. O microorganismo invasor mais comum é a Escherichia coli, detectada em cerca de $80 \%$ a $90 \%$ das infecções bacterianas agudas não-complicadas das vias urinárias. O Staphylococcus saprophyticus pode ser responsável por $10 \%$ a $20 \%$ dos casos em mulheres jovens adultas, sendo descrito como a segunda causa mais freqüente de ITU nessas pacientes, enquanto que outras bactérias gram-positivas são relativamente raras, podendo incluir estreptococos dos grupos B e D. Nas infecções complicadas, na presença de anormalidade anatômica ou cateterização crônica, o espectro de bactérias infectantes é maior do que nos casos não-complicados. Verifica-se quase sempre a presença de $E$. coli, porém muitas espécies de outros bastonetes gram-negativos (por exemplo, Klebsiella, Proteus, Enterobacter e Pseudomonas), enterococos e estafilococos também são comuns. Em muitos casos são identificadas duas ou mais espécies, e as bactérias são quase sempre resistentes aos agentes antimicrobianos administrados ${ }^{(4)}$.

O diagnóstico de uma infecção do trato urinário é sempre feito em bases clínicas e laboratoriais ${ }^{(19)}$. As técnicas laboratoriais mais empregadas incluem a análise qualitativa e a cultura da urina (urocultura), sendo esse último o método considerado padrão-ouro para o diagnóstico de ITU ${ }^{(20,}$ 23). A urocultura quantitativa é o exame mais importante para o diagnóstico de uma infecção urinária, pois não apenas indica a ocorrência de multiplicação bacteriana no trato urinário, mas também permite o isolamento do agente causal e o estudo de sua sensibilidade frente aos antimicrobianos. Habitualmente, considera-se positiva a presença de número igual ou superior a cem mil $\left(>10^{5}\right)$ unidades formadoras de colônias bacterianas por mililitro de urina adequadamente coletada. Entretanto esse método apresenta desvantagens, como a demora no diagnóstico e o alto custo(17).

Um teste ideal deve apresentar alta acurácia, requerer limitada perícia técnica e ter baixo custo, possibilitando um rápido diagnóstico em pacientes de alto risco. Um exemplo seria o teste com tiras reagentes, em que somente nitrito e esterase leucocitária - e não proteínas e sangue - mostram razoável precisão e exatidão, em comparação com a cultura quantitativa ${ }^{(7)}$. O teste da esterase leucocitária é um meio rápido de verificar a presença de piúria; um teste positivo correlaciona-se com oito a dez leucócitos por campo de grande aumento(3). A associação entre nitrito urinário e ITU foi inicialmente relatada em $1914^{(12)}$. O teste de nitrito positivo requer a presença de nitratos na urina, bactérias em quantidades suficientes capazes de converter nitratos em nitritos e condições adequadas (isto é, tempo suficiente) para que essa conversão ocorra ${ }^{(13,18)}$. Os testes que utilizam tiras reagentes detectam significativamente mais infecções por bactérias gram-negativas do que por espécies gram-positivas, uma vez que o teste de nitrito não revela a presença de patógenos gram-positivos em muitos casos ${ }^{(21)}$. A conversão de nitrato em nitrito na urina por cocos gram-positivos ainda não está bem estabelecida. Murray et al.(16) relatam que essa conversão é determinada apenas para estafilococos coagulase-negativos, sendo na maioria das vezes positiva, e para Staphylococcus saprophyticus, cuja conversão é sempre negativa. Algumas espécies de cocos, como, por exemplo, os enterococos, são incapazes de reduzir o nitrato urinário em nitrito e apresentam alta resistência às drogas comumente utilizadas para tratamento de ITU(12).

Dessa forma, o objetivo do presente trabalho foi explorar a microbiologia e a freqüência de ITU por cocos em pacientes sintomáticos, relacionando com o resultado da prova de nitrito urinário, a fim de evidenciar se existem diferenças quanto ao perfil epidemiológico dos pacientes e à suscetibilidade aos antimicrobianos. 


\section{Material e método}

Um estudo retrospectivo foi conduzido na Secretaria de Saúde do município de Maringá-PR, no período de abril de 2004 a março de 2005. Esse estudo foi baseado na análise dos resultados de exames de urina de pacientes atendidos nas Unidades Básicas de Saúde (UBSs) de Maringá e realizado pelo Laboratório Municipal de Análises Clínicas do município. Os pacientes incluídos apresentaram um ou mais dos seguintes sinais e sintomas: disúria, freqüência urinária, dor abdominal baixa e febre. Todos eles tinham resultado de cultura de urina positiva, com teste de sensibilidade e urinálise disponíveis. Indivíduos menores de 18 anos foram excluídos. Esse estudo foi aprovado pelo Comitê Permanente de Ética em Pesquisa Envolvendo Seres Humanos (COPEP) da Universidade Estadual de Maringá (UEM) (Registro no 228/2004, Parecer o 032/2005, Cl no 031/2005).

As amostras foram obtidas no laboratório através da coleta da primeira urina da manhã, em recipientes estéreis, utilizando-se a técnica do jato médio de micção espontânea. Após a coleta, o material foi encaminhado ao Setor de Microbiologia, onde foi registrado e semeado em placas contendo meio cistina lactose eletrólitos deficiente (CLED) (Merk $^{\circledR}$ ), utilizando-se alça calibrada de $1 \mu$ l, e incubado a $37^{\circ} \mathrm{C}$ por 24 horas. A amostra foi então encaminhada ao Setor de Urinálise para realização do parcial de urina. O exame foi realizado através do uso de tiras reagentes Combur $^{10}$ Test ${ }^{\circledast}$ UX (Roche Diagnostics), de acordo com as instruções do fabricante. Os testes para nitrito e esterase leucocitária foram considerados positivos quando a área reagente da tira apresentava coloração rósea e $1+$ ou $2+$, respectivamente. O exame microscópico do sedimento urinário foi feito após centrifugação a $1.500 \mathrm{rpm}$ por 5 minutos. O número de leucócitos na amostra de urina centrifugada foi determinado em câmara K-Cell ${ }^{\circledR}($ Kima), sendo piúria definida como $=10$ leucócitos $/ \mathrm{mm}^{3}$ de urina. Após contagem das colônias na urocultura, os microrganismos foram semeados em placas negative urine combo (NUC-2) para bacilos gram-negativos e positive combo (PC-21) para cocos gram-positivos, para identificação bioquímica e antibiograma, e a leitura foi realizada pelo aparelho de automação MicroScan ${ }^{\circledast}$ (Dade Behring, Inc., West Sacramento, Calif.). Essas placas contêm também a prova de nitrato, confirmando o resultado de nitrito urinário realizado nas tiras reagentes. Para este trabalho, as culturas foram consideradas positivas quando havia $>10^{5}$ unidades formadoras de colônia (UFC) de um único microrganismo por mililitro de urina.

Dados demográficos, sintomatologia, co-morbidades e tratamento antimicrobiano dos pacientes cuja cultura de urina foi positiva para cocos foram extraídos dos prontuários existentes nas UBSs municipais através de revisão retrospectiva. Os pacientes foram divididos em dois grupos com base no resultado de nitrito urinário (positivo ou negativo). Os resultados de esterase leucocitária, hematúria e teste de suscetibilidade a antimicrobianos, bem como os dados retirados dos prontuários, foram comparados em cada grupo para descrição do perfil epidemiológico dos pacientes.

\section{Análise estatística}

As análises foram realizadas utilizando-se o programa Biostat ${ }^{\oplus}$ 2.0. As variáveis foram comparadas através do $\chi^{2}$ ou teste de Fisher. Diferenças entre as variáveis contínuas foram comparadas com o teste $t$ de Student. O $p$ foi considerado significativo quando $<0,05$.

\section{Resultados}

Amostras de urina de 3.426 pacientes foram coletadas e analisadas pelo Laboratório Municipal de Análises Clínicas de Maringá no período estudado. Um total de 448 (13,1\%) foi positivo para cultura. As espécies bacterianas causadoras de ITU estão sumarizadas na Tabela 1. Em 388 casos $(86,6 \%)$ houve crescimento de bacilos gram-negativos (BGN) e em 60 (13,4\%), de cocos gram-positivos (CGP). Escherichia coli foi o agente mais freqüente $(61,2 \%), \mathrm{e}$, com relação aos CGP, o Enterococcus faecalis foi o mais encontrado (2,9\%). A idade média dos pacientes com resultado de cultura positiva foi 51,7 anos (variando de 18 a 94 anos). Mulheres representaram $82,6 \%$ dos pacientes $(n=370)$ e homens, $17,4 \%(n=78)$. Entre as culturas positivas para cocos, a idade média dos pacientes foi 53,4 anos (variando de 18 a 94 anos), sendo que as mulheres representaram $85 \%$ dos pacientes $(n=51)$ e os homens, $15 \%(n=9)$.

A Tabela 2 mostra a freqüência dos CGP nos casos de infecção urinária com resultado de nitrito positivo e negativo. Um número maior de isolados de $E$. faecalis e de S. epidermidis demonstrou ser não-redutor de nitrato (NIT), enquanto que, em relação aos isolados de S. aureus, a maioria mostrou-se redutor de nitrato (NIT+). Streptococcus agalactiae e $\mathrm{S}$. saprophyticus foram isolados exclusivamente em casos de nitrito negativo.

Os dados demográficos de pacientes com resultado de nitrito positivo versus aqueles com nitrito negativo foram similares no que se refere à idade e à distribuição por sexo. Não houve diferença significativa com relação à 


\section{Tabela 1 Bactérias causadoras de infecção do trato urinário na população estudada}

\begin{tabular}{lcc}
\hline Organismo & Número & $\%$ \\
Bacilos gram-negativos (BGN) & 388 & 86,6 \\
Escherichia coli & 274 & 61,2 \\
Klebsiella pneumoniae & 31 & 6,9 \\
Enterobacter cloacae & 16 & 3,5 \\
Proteus mirabilis & 13 & 2,9 \\
Citrobacter koseri & 12 & 2,7 \\
Outros & 42 & 9,4 \\
& & \\
Cocos gram-positivos (CGP) & 60 & 13,4 \\
Enterococcus faecalis & 13 & 2,9 \\
Staphylococcus epidermidis & 12 & 2,7 \\
Streptococcus agalatiae & 11 & 2,5 \\
Staphylococcus saprophyticus & 10 & 2,2 \\
Staphylococcus aureus & 9 & 2 \\
Outros & 5 & 1,1 \\
Total & 448 & 100 \\
\hline
\end{tabular}

Outros: Citrobacter freundii, Pseudomonas aeruginosa, Enterobacter aerogenes, Escherichia fergusonii, Morganella morganii, Acinetobacter baumannii, Enterobacter agglomerans, Serratia marcescens, Serratia liquefaciens, Cedecea sp., Providencia rettgeri, Providencia stuartii, Klebsiella oxytoca e Alcaligenes xylosoxidans (BCN); Staphylococcus xylosus, Staphylococcus warneri, Enterococcus faecium, Staphylococcus simulans e Staphylococcus auricularis (CGP).

\section{Tabela 2 urinário positivo e negativo}

\begin{tabular}{lccc}
\hline & Nitrito $+(n=16)$ & Nitrito- $(n=44)$ & Total $(n=60)$ \\
Organismo & Número $(\%)$ & Número $(\%)$ & Número $(\%)$ \\
Enterococcus faecalis & $2(12,5)$ & $11(25)$ & $13(21,7)$ \\
Staphylococcus epidermidis & $5(31,4)$ & $7(15,9)$ & $12(20)$ \\
Streptococcus agalactiae & 0 & $11(25)$ & $11(18,3)$ \\
Staphylococcus saprophyticus & 0 & $10(22,7)$ & $10(16,7)$ \\
Staphylococcus aureus & $6(37,5)$ & $3(6,8)$ & $9(15)$ \\
Staphylococcus xylosus & $1(6,2)$ & 0 & $1(1,7)$ \\
Staphylococcus warnerii & $1(6,2)$ & 0 & $1(1,7)$ \\
Staphylococcus simulans & 0 & $1(2,3)$ & $1(1,7)$ \\
Staphylococcus auricularis & $1(6,2)$ & 0 & $1(1,7)$ \\
Enterococcus faecium & 0 & $1(2,3)$ & $1(1,7)$ \\
\hline
\end{tabular}

positividade para esterase leucocitária, piúria e hematúria. A análise estatística de co-morbidades dos pacientes revelou diferença significativa na proporção de pacientes com hipertensão ( $31,3 \%$ com nitrito positivo versus $4,5 \%$ com nitrito negativo, $p=0,0116)$. Os pacientes com resultado de nitrito positivo relataram febre em proporção maior que os com nitrito negativo $(75,0 \%$ versus $43,2 \%$, respectivamente; $p=0,0414)$. As bactérias NIT+ exibiram maior resistência à ciprofloxacina $(43,8 \%)$ em comparação com as NIT- $(13,6 \%)$ (Tabela $\mathbf{3})$. 


\section{Tabela 3}

\section{Comparação entre as características dos pacientes e os resultados da urinálise, da cultura e da} sensibilidade a antimicrobianos dos casos de infecção urinária por cocos gram-positivos NIT+

\begin{tabular}{lccc} 
Características dos pacientes & NIT+ $(n=16)$ & NIT- $(n=44)$ & $p$ \\
Média de idade em anos (variação) & $59,2(19-92)$ & $51,3(18-94)$ & 0,1993 \\
Mulheres (\%) & $13(81,3)$ & $38(86,4)$ & 0,6882 \\
Co-morbidade & & & \\
$\quad$ Diabetes & $2(12,5)$ & $2(4,5)$ & 0,5654 \\
$\quad$ Hipertensão & $5(31,3)$ & $2(4,5)$ & 0,0116 \\
$\quad$ Acidente vascular cerebral & $1(6,3)$ & 0 & nd \\
Sinais e sintomas ${ }^{1}$ & $12(75)$ & $19(43,2)$ & 0,0414 \\
$\quad$ Febre & $13(81,3)$ & $36(81,8)$ & 0,9949 \\
$\quad$ Disúria & $12(75)$ & $24(54,5)$ & 0,2341 \\
Frequiência urinária & $15(93,8)$ & $34(77,3)$ & 0,2586 \\
$\quad$ Dor abdominal baixa & $11(68,8)$ & $37(84,1)$ & 0,273 \\
Positividade para esterase leucocitária & $16(100,0)$ & $37(84,1)$ & 0,1733 \\
Piúria & $8(50)$ & $21(47,7)$ & 1 \\
Hematúria & & & \\
Sensibilidade à ciprofloxacina & & $26(59,1)$ & 0,8435 \\
$\quad$ Sensíveis & $9(56,2)$ & $6(13,6)$ & 0,0287 \\
Resistentes & $7(43,8)$ & 7 & 0,1534 \\
Número de espécies bacterianas & 6 & 7 & \\
\hline
\end{tabular}

${ }^{1}$ Não obtivemos o resultado de um paciente com infecção urinária por CGP redutor de nitrato.

${ }^{2}$ Não testada para Streptococcus agalactiae.

${ }^{3}$ Isoladas por cultura quantitativa.

nd: não determinado.

Os antimicrobianos mais prescritos pelos médicos para a população em estudo foram a ciprofloxacina e a norfloxacina. A Figura mostra o perfil de resistência aos antimicrobianos nos pacientes com infecção por cocos. De maneira geral, os isolados NIT+ exibiram maior resis- tência a estes dois antimicrobianos do que os isolados NIT-. S. aureus NIT+ mostraram grande resistência a ciprofloxacina (66,7\%) e norfloxacina (66,7\%), enquanto os isolados NIT- exibiram $100 \%$ de sensibilidade a esses antimicrobianos.

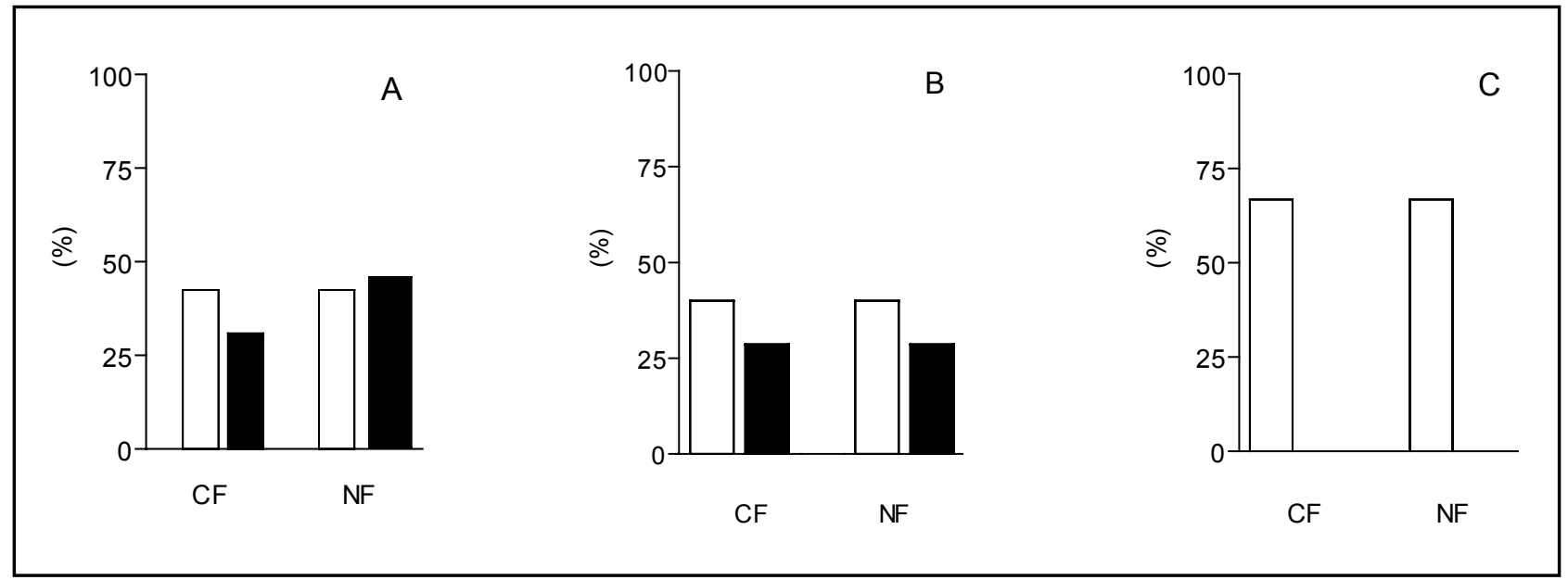

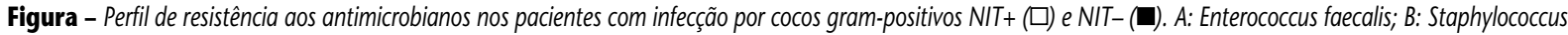
epidermidis; C: Staphylococcus aureus. CF: Ciprofloxacina; NF: Norfloxacina. 


\section{Discussão}

Neste trabalho, das 3.426 amostras de urina coletadas e analisadas, apenas $448(13,1 \%)$ foram positivas para cultura. Os pacientes procuraram as UBSs do município por apresentarem disúria, freqüência urinária, dor abdominal baixa e/ou febre. Os exames de urinálise e urocultura foram solicitados pelos médicos devido a tais sintomas sugestivos de infecção do trato urinário. Entretanto sintomatologia que sugere infecção urinária pode ocorrer na dependência de inúmeros outros fatores, principalmente na mulher, como colpites, vaginites, uretrites, etc. ${ }^{(17)}$.

Do total de amostras de urina positivas para cultura, em $60(13,4 \%)$ casos foram isolados CGP, como observado na Tabela 1, estando de acordo com outros estudos que demonstram freqüência de CGP em torno de $7 \%-21 \%(1,15)$. As mulheres representaram $85 \%$ dos pacientes $(n=51)$. Infecções que acometem o trato urinário feminino, chamadas de não-complicadas, compreendem problemas que vão desde cistite até infecções urinárias recorrentes, que ocorrem principalmente em jovens sexualmente ativas. Esse tipo de infecção é dez a 20 vezes mais freqüente em mulheres do que em homens. Tal fato se deve à formação anatômica da genitália externa, assim como ao comprimento da uretra feminina, que favorece a ascensão das enterobactérias que colonizam o intróito vaginal para o interior da bexiga ${ }^{(2)}$.

O teste das tiras reagentes para bacteriúria (teste de Greiss) fundamenta-se na medida indireta de bactérias redutoras do nitrato a nitrito. Segundo Murray et al. (16), para a maioria dos $B G N$, incluindo $E$. coli, essa conversão ocorre em 99\%-100\% dos casos. Já para CGP, ainda não está bem estabelecida, dependendo muito da espécie bacteriana. Franz e Hörl(8) relataram que a utilidade desse teste está restrita às enterobactérias, para as quais o resultado é positivo. Entretanto, em muitos casos, encontramos resultado de nitrito positivo e infecção por CGP.

Os pacientes geralmente são tratados com base na suspeita clínica. Uma prática comum entre os médicos é relacionar sintomatologia de ITU com resultado de nitrito positivo na tira reagente com infecção por BGN. Assim, torna-se interessante estudar esses casos de infecção por CGP, com nitrito positivo ou negativo, para evidenciar se existiriam diferenças quanto ao comportamento da bactéria e à resposta do hospedeiro. Neste trabalho não houve diferença significativa na maioria dos parâmetros avaliados (Tabela 3). Entretanto encontramos mais casos de hipertensão e febre entre os pacientes com infecção por CGP NIT+.
As bactérias isoladas nos casos de nitrito positivo exibiram maior resistência à ciprofloxacina do que aquelas isoladas em casos de nitrito negativo.

Larson et al. ${ }^{(12)}$ avaliaram se o nitrito urinário poderia ser usado para guiar a terapia antimicrobiana. Uma vez que, segundo eles, os enterococos não são capazes de reduzir o nitrato e apresentam alta resistência ao trimetoprim-sulfametoxazol (TMP-SMX), os casos com nitrito positivo excluiriam infecção por essas bactérias e poderiam ser tratados com esse antimicrobiano. Já os casos com nitrito negativo seriam tratados com outro antimicrobiano desde o início. No entanto, não evidenciaram diferença significativa entre a proporção de isolados NIT+ e NIT- sensíveis ao TMP-SMX, já que os enterococos podem crescer em culturas mistas.

As vantagens em utilizar o nitrito urinário são o baixo custo, a rapidez com que os resultados tornam-se disponíveis e sua habilidade para categorizar os pacientes em dois grupos distintos, nitrito positivo ou negativo ${ }^{(12)}$. A enzima nitrato redutase, que reduz o nitrato a nitrito, está presente nos coliformes, mas não em outras bactérias como S. saprophyticus e enterococos ${ }^{(9)}$. Entretanto, neste trabalho, encontramos dois casos de infecção por Enterococcus faecalis NIT+ (Tabela 2). Um desses isolados exibiu sensibilidade a todos os antimicrobianos testados, enquanto o outro foi resistente a ciprofloxacina e norfloxacina, entre outros. As duas pacientes, uma de 79 e outra de 19 anos, apresentavam todos os sinais e sintomas sugestivos de ITU e não faziam uso de nenhum medicamento. Holloway et al.(10) relataram que $5,3 \%$ de suas amostras com cultura pura para enterococos produziram nitrito positivo. $\mathrm{O}$ tempo que a urina permanece na bexiga pode influenciar a positividade do teste de nitrito. Uma alta porcentagem de resultados positivos tem sido observada quando pelo menos 4 horas se passam desde a última micção. Por essa razão, recomenda-se que todas as provas de nitrito sejam feitas com a primeira urina da manhã, que permanece na bexiga durante várias horas ${ }^{(22)}$.

Ocorreram mais casos de $S$. aureus nitrito positivo que nitrito negativo. Os isolados NIT+ mostraram grande resistência a ciprofloxacina $(66,7 \%)$ e norfloxacina $(66,7 \%)$, enquanto que os isolados NIT- exibiram $100 \%$ de sensibilidade a esses antimicrobianos (Figura).

Uma ampla variedade de agentes antimicrobianos é usada na profilaxia e no tratamento de infecções do trato urinário, sendo que um grave problema tem sido a resistência crescente de patógenos aos antibióticos convencionais. Em adição, a administração oral de agentes antimicrobianos pode perturbar a microflora intestinal normal, resultando 
em diarréia e infecções oportunistas ${ }^{(5)}$. A escolha dos agentes antimicrobianos depende das políticas locais e dos padrões de resistência da população. As drogas de primeira linha sugeridas incluem TMP-SMX, cefalexina, nitrofurantoína e ciprofloxacina $a^{(9)}$. Para os pacientes com resultado de cultura positiva para cocos gram-positivos, a ciprofloxacina foi prescrita em $46,7 \%$ dos casos, mostrando ser a droga de escolha pelos médicos atendentes das UBSs de Maringá. Entretanto, como pôde ser observado na Tabela 3, as bactérias redutoras de nitrato exibiram maior resistência a ciprofloxacina em comparação com as que não o fazem $(43,8 \%$ versus $13,6 \%$, respectivamente, $p=0,0287)$. Um estudo mais aprofundado desses microrganismos, juntamente com uma amostragem maior, poderia fornecer informações importantes sobre mecanismos básicos de ação antimicrobiana e desenvolvimento de resistência. Médicos e microbiologistas devem estar preparados para o desafio do reconhecimento de novos e evoluídos patógenos bacterianos.

\section{Conclusões}

A importância clínica das infecções do trato urinário não decorre apenas de sua elevada prevalência, mas sim das conseqüências e complicações que podem produzir. O tratamento bem-sucedido dessas infecções depende essencialmente do diagnóstico clínico e bacteriológico correto, bem como do acompanhamento do doente para verificar a ocorrência de recidiva da infecção. Apesar de as ITUs causadas por CGP serem menos prevalentes que as causadas por BGN, em muitos casos o tratamento pode não ser adequado, trazendo prejuízos à saúde do paciente.

Este trabalho procurou demonstrar se existiriam diferenças quanto ao comportamento dos CGP nos casos de ITU com resultado de nitrito positivo e negativo. Alterações significativas foram evidenciadas com relação a alguns sintomas, co-morbidades e resistência aos antimicrobianos. Entretanto, devido ao pequeno número de amostras, torna-se difícil qualquer conclusão. Não podemos relacionar a resistência aos antimicrobianos com a capacidade da bactéria em reduzir o nitrato. Porém, este estudo preliminar mostrou que a avaliação dos sinais clínicos e sintomas, juntamente com o resultado de nitrito urinário na tira reagente, poderia auxiliar o médico na tomada de decisões importantes. Pesquisas utilizando biologia molecular e uma amostragem maior seriam interessantes para um melhor entendimento sobre as infecções do trato urinário por cocos gram-positivos.

\section{Referências}

I. AGUIRRE-AVALOS, G. et al. Asymptomatic bacteriuria and inflammatory response to urinary tract infection of elderly ambulatory women in nursing homes. Arch Med Research, v. 30, p. 29-32, 1999.

2.ARAÚJO, M.A.T.; ROCHA-FILHO, M.A.; IMBROISI, M.A. Infecção do trato urinário em mulheres. Int Braz J Urol, v. 29, p. 25-9, 2003.

3. BARROS, E. et al. Nefrologia: rotina, diagnóstico e tratamento. 2. ed. Porto Alegre: ArtMed, 1999.

4. BROOKS, G.F.; BUTEL, J.S.; MORSE, S.A. Microbiologia médica. 21. ed. Rio de Janeiro: Guanabara-Koogan, 2000.

5. CARLSSON, S. et al. In vitro evaluation of a new treatment for urinary tract infections caused by nitrate-reducing bacteria. Antimicrob Agents Chemother, v. 47, n. 12, p. 37I3-8, 2003.

6. CORRÊA, L.A.; CANALINI, A.F.; MATHEUS, W.E. Etiologia das infecções do trato urinário. Int Braz J Urol, v. 29, p. 7-10, 2003.

7. DEVILLÉ,W.L.J.M. et al.The urine dipstick test useful to rule out infections. A meta-analysis of the accuracy. BMC Urol, v. 4, n. I, p. 4-17, 2004.

8. FRANZ, M.; HÖRL, W.H. Common errors in diagnosis and management of urinary tract infection. I: Pathophysiology and diagnostic techniques. Nephrol Dial Transplant, v. I4, p. 2746-53, 1999

9. GRAHAM, J.C.; GALLOWAY, A. The laboratory diagnosis of urinary tract infection. J Clin Pathol, v. 54, p. 91।-9, 200।.

10. HOLLOWAY, J.; JOSHI, N.; O'BRYAN, T. Positive urine nitrite test: an accurate predictor of absence of pure enterococcal bacteriuria. South Med J, v. 93, n. 7, p. 68I-2, 2000.

I I. KUNIN, C.M. Urinary tract infections. 5. ed. Baltimore: Williams \&Wilkins, 1997

12. LARSON, M.J. et al. Can urinary nitrite results be used to guide antimicrobial choice for urinary tract infection? J Emerg Med, v. I5, n. 4, p. 435-8, 1997.

13. LOHR, J.A. et al. Making a presumptive diagnosis of urinary tract infection by using a urinalysis performed in an on-site laboratory.J Pediatr, v. 122, p. 22-5, 1993.

I4.MARANGONI, D.V.; MOREIRA, B.M. Doenças infecciosas: conduta, diagnóstico e terapêutica. Rio de Janeiro: Guanabara-Koogan, 1994.

15. McNAIR, R.D. et al. Evaluation of the centrifuged and Gramstained smear, urinalysis, and reagent strip testing to detect asymptomatic bacteriuria in obstetric patients. Am J Obstet Gynecol, v. I 82, n. 5, p. 1076-9, 2000. 
16. MURRAY, P.R. et al. Manual of clinical microbiology. 7. ed. Washington: ASM Press, 1999.

17. RIELLA, M.C. Princípios de nefrologia e distúrbios hidroeletrolíticos. 4. ed. Rio de Janeiro: Guanabara-Koogan, 2003.

I8. ROBERTSON,A.W.; DUFF, P.The nitrite and leukocyte esterase tests for the evaluation of asymptomatic bacteriuria in obstetric patients. Obstet Gynecol, v. 7I, n. 6, p. 878-8I, 1988.

19. ROCHA, L.C.A.; CARVALHAL, G.F.; MONTI, P.R. Exames complementares na infecção do trato urinário. Int Braz J Urol, v. 29, p. 15-20, 2003.
20. SANTOS, S.R.S.R.; AMADO, C.A.B.; ASSEF, S.M.C. Infecções urinárias. Arq Ciênc Saúde Unipar, v. 3, n. I, p. 43-50, 1999.

2I.SEMENIUK, H.; CHURCH, D. Evaluation of the leukocyte esterase and nitrite urine dipstick screening tests for detection of bacteriuria in women with suspected uncomplicated urinary tract infections. J Clin Microbiol, v. 37, n. 9, p. 305।-2, 1999.

22. STRASINGER, S.K. Uroanálise e fluidos biológicos. 3. ed. São Paulo: Editorial Premier, 1998.

23. ZAMAN,A.B. et al. Disappointing dipstick screening for urinary tract infection in hospital inpatients. J Clin Pathol, v. 5I, p. $47 \mid-2,1998$ 